\title{
Rätselhafte Artefakte. Leo Perutz und das unzuverlässige Erzählen
}

\author{
Hans-Harald Müller
}

Zusammenfassung Nach einem knappen Rückblick auf die Begriffsgeschichte des unzuverlässigen Erzählens wird das Romanwerk von Leo Perutz im Hinblick auf Vorkommen und spezifische Verwendungen des unzuverlässigen Erzählens untersucht. Auffällig ist in diesem Kontext, dass Perutz seit 1933 keinen Gebrauch mehr von dieser Erzählkonzeption macht. Die Hypothese, dass dieser Sachverhalt auf eine - in der deutschen Literaturgeschichte des 20. Jahrhunderts oft konstatierte - ,Abschwächung der Moderne' seit Ende der 1920er Jahre zurückzuführen ist, lässt sich jedoch nicht erhärten. Beim gegenwärtigen Stand der Forschung kann das unzuverlässige Erzählen weder einer spezifischen poetischen Konzeption noch einer begrenzten literarhistorischen Epochen zugeordnet werden.

\section{Das unzuverlässige Erzählen in Perutz' Romanen. Ein Überblick}

Aus einer analytisch aufgeklärten Rezeptionsgeschichte des Begriffs des unzuverlässigen Erzählens könnten die humanities einiges über Nachhaltigkeit und Folgenabschätzung für die Klärung und Verwendung von Begriffen lernen. Der Begriff des unzuverlässigen Erzählens wurde in einer epistemisch recht unklaren Situation in einer unklaren Fassung geprägt, in der Folgezeit mit einer Fülle weitreichender Leistungsanforderungen überschüttet und in Anwendungen ausgebeutet. Der Effekt war, dass im Jahre 2015 die emphatische Forderung erhoben wurde, „die Kategorie der unreliable narration aufzugeben“ (Ohme 2015, S. 209 f.). Inzwischen haben sich die Wogen vielleicht etwas geglättet; man kann

H.-H. Müller $(\varangle)$

Institut für Germanistik, Universität Hamburg,

Hamburg, Deutschland

E-Mail: harrym@uni-hamburg.de 
sehen, dass die strukturalistischen, die analytischen und pragmatischen Arbeiten am meisten zur Begriffsklärung, die kulturalistischen am meisten zur Begriffsverbreitung und -verwendung beigetragen haben. Die analytischen Begriffsexplikationen besitzen den Vorzug der Klarheit; sie unterstreichen zugleich, dass der Nutzen des Begriffs weit weniger ein klassifikatorischer denn ein heuristischer (vgl. Kindt und Köppe 2011, S. 88) ist, dessen aufschließende Kraft sich in der Interpretation und der Bestimmung der Funktion (vgl. dazu etwa Aumüller 2018) des unzuverlässigen Erzählens erweisen muss.

Leo Perutz' Romane sind zu lang, um sie zu ausführlich zu zitieren, und stilistisch zu elaboriert, um sie zusammenzufassen. Der folgende Überblick über Perutz Romanwerk soll lediglich zeigen, dass das Verfahren narrativer Unzuverlässigkeit eines von verschiedenen Mitteln sein kann, um charakteristische Ziele von Perutz' Romanpoetik zu erfassen. Im ersten Roman, Die dritte Kugel, gibt es noch keinen unzuverlässigen Erzähler. Perutz fordert den Leser hier auf, sich eine Rahmen- und eine Binnenhandlung vorzustellen, deren einzige tragende Verbindung eine Metalepse herstellt: die dritte Kugel eben, die nach dem Durchschlagen der Grenze zwischen intradiegetischer und extradiegetischer Welt den Binnenerzähler trifft. Was für Die dritte Kugel charakteristisch ist, gilt cum grano salis für alle Perutz-Romane: Sie bedienen sich ausgeklügelter plot-Konstruktionen, sie haben meist am zeitgenössischen Diskurs um Identität und Identitätsverlust des Individuums $^{1}$ (vgl. Titzmann 1989, 2002) teil und sie machen, indem sie vom Erzählten auf das Erzählen selbst verweisen, stets den Konstruktionscharakter der Texte bewusst; sie sind also - auch - metafiktionale Romane.

Auf der Suche nach einem unzuverlässigen Erzähler wird man in Perutz' zweitem Roman fündig, Zwischen neun und neun, einem Gegenwartsroman. Sein Held ist der Wiener Student Stanislaus Demba, der sich morgens um neun, bereits in Handschellen, durch den kühnen Sprung von einem Hausdach dem Zugriff der Polizei entzieht, sich aufrafft und tagsüber verzweifelt versucht, Geld für eine Reise mit seiner nicht recht treuen Geliebten Sonja aufzutreiben und die Handschellen loszuwerden, um schließlich um neun Uhr abends, am Boden zerschmettert, zu sterben - die Erlebnisse zwischen neun und neun waren nur eine Traumvision.

Bis zum 8. Kapitel muss gerätselt werden, was es mit Dembas Händen auf sich hat, denn den Lesern werden seine Handschellen vorenthalten. Vom 9. Kapitel an wächst die Spannung, ob Demba das Reisegeld zusammenbekommen und von seinen Handschellen befreit werden wird. Die Schlusswendung bietet die Überraschung, dass Dembas Leben zwischen neun und neun eine Sterbevision ${ }^{2}$ war. Die Reaktion der Leser ist meist jedoch weniger von Betroffenheit über den Tod des Helden geprägt als von Bewunderung darüber, wie viel Leben Demba aus den wenigen Sekunden seines Sterbens herausgeholt hat.

\footnotetext{
${ }^{1}$ Vgl. auch den Abschnitt „Vergessen, Erinnerung und Erzählen bei Leo Perutz“, Orosz (2016, S. 305-331).

${ }^{2}$ Zur Gattung s. die luzide Untersuchung von Lamping (1987, S. 71-95).
} 
Unzuverlässig ist der heterodiegetische Erzähler in zweierlei Hinsicht: Bei sonst detaillierter Beschreibung des Protagonisten informiert er den Leser viele Kapitel lang nicht über Dembas Handschellen, und er enthält dem Leser bis zur Schlusswendung die entscheidende Information über den Realitätsstatus des Erzählten vor. ${ }^{3}$ Der final twist bewirkt aber weit mehr als einen Überraschungseffekt wie etwa in Ambrose Bierces Erzählung An Occurrence at Owl Creek Bridge. Er führt nämlich für den Interpreten zu einer ähnlich aporetischen Situation wie die metaleptische Verbindung zwischen Binnen- und Rahmenhandlung in der Dritten Kugel. Die Geschehnisse zwischen neun und neun können aufgrund der Versicherung des Erzählers nicht als reale Ereignisse gelten - sie können aufgrund der für Traumerzählungen geltenden Erzählkonventionen aber auch nicht als geträumt gelten: $\mathrm{Zu}$ offensichtlich werden die Geschehnisse nicht in interner Fokalisierung vom Wahrnehmungs- und Wissensstandpunkt Dembas berichtet, sondern von einem heterodiegetischen Erzähler. ${ }^{4}$ Der im Zweifel verbleibende ontologische Status des Erzählten verweist auf den Konstruktionscharakter eines Romans, der zum einen alle Effekte einer realistischen analytischen Rätselerzählung ${ }^{5}$ ausbeutet, den Leser zugleich aber auffordert, sich eine Erzählung vorzustellen, die die Konventionen dieser Erzählung innerfiktional infrage stellt.

Perutz' dritter Roman, Der Marques de Bolibar, ist erneut ein historischer Roman. Er weist motivisch einige Ähnlichkeiten mit der Dritten Kugel auf und ist genauso anspruchsvoll konzipiert. Die von einem anonymen Herausgeber um 1870 gefundenen und edierten Memoiren des Rittergutsbesitzers von Jochberg sollen ein ,dunkles und vorher niemals aufgeklärtes Kapitel der vaterländischen Kriegsgeschichte“ (Perutz 2006, S. 8) erhellen, den Untergang zweier Rheinbundregimenter im spanischen Befreiungskrieg gegen Napoleon. Die Vernichtung des Regiments „Nassau“, behaupten die Memoiren, sei „von seinem Offizierskorps mit vollem Bewußtsein, ja beinahe planmäßig herbeigeführt worden“ (Perutz 2006, S. 9). ${ }^{6}$ Die Spannung richtet sich darauf, wie dieser nicht allein in der Kriegs-, sondern auch in der Literaturgeschichte aller Zeiten wohl einzig dastehende und höchst unwahrscheinliche Fall sich zutrug, zufällig oder auf übernatürliche Weise schicksalhaft. ${ }^{7}$ Das nun soll die in Ich-Form erzählte

\footnotetext{
${ }^{3}$ In beiden Fällen handelt es sich um täuschendes Erzählen mit geschlossener Funktion, nach Aumüller (2018, S. 134-138).

${ }^{4}$ Beispiele: der Träumende wendet sich an das Lesepublikum; das Traumgeschehen wird chronologisch geschildert, nicht in Zeitsprüngen etc. Demba tritt als erzählte Figur auf, Dembas Verhalten wird vom Erzähler kritisch beurteilt, der Erzähler berichtet über Situationen, in denen Demba nicht anwesend ist etc.

${ }^{5} \mathrm{Zu}$ dieser für Perutz charakteristischen Struktur s. Martínez (2002, S. 107-129).

${ }^{6}$ Dort kursiv.

${ }^{7}$ Die Frage, ob der Untergang auf (1) empirisch-kausale oder (2) übernatürliche Weise herbeigeführt wird, halte ich trotz zahlreicher Indizien für (2) im Sinne von (1) für entschieden. Martínez möchte für die abschließende Verwandlung Jochbergs in den Marques ebenfalls eine übernatürliche Erklärung in Anspruch nehmen. Die Verwandlung Jochbergs aber gehört nicht zu der im Vorwort markierten erklärungsbedürftigen Ereigniskette, die den Untergang der Rheinbundregimenter betrifft. Martínez übersieht, dass, selbst wenn für die Verwandlung Jochbergs
} 
Binnengeschichte des Leutnant von Jochberg erhellen. Zur Erklärung des Untergangs der Regimenter sind, systematisch betrachtet, vier Ereignisse notwendig.

1. Der Marques de Bolibar entwickelt bei einem Treffen mit den Insurgenten den Plan eines bewaffneten Aufstands in dem Ort La Bisbal; der Aufstand soll durch drei Signale ausgelöst werden.

2. Das Treffen wird von einem deutschen Offizier belauscht, der seinen Kameraden von den vereinbarten Signalen berichtet: Sie wissen also, woher der Untergang droht.

3. Der als Maultiertreiber verkleidete Marques de Bolibar gerät in die Gefangenschaft der deutschen Offiziere und wird von ihnen erschossen - vor seinem Tod aber hat er ihnen einen heiligen Eid abgenommen, dass sie die Aufgaben erledigen werden, derentwegen er in die Stadt gekommen war, nämlich die Signale zum Aufstand zu geben. Nur der Leutnant Jochberg erkennt, dass er und seine Kameraden in der Gestalt des Maultiertreibers den Marques de Bolibar erschossen und er ahnt, dass sie mit ihrem Eid den eigenen Untergang besiegelt haben.

4. Die Offiziere lösen die drei Signale auf höchst unterschiedliche Weise aus: das erste strategisch, das zweite spontan, das dritte trägt Jochberg eher zufällig ins feindliche Lager. Auf diese Weise wird der Plan des toten Marques realisiert: Der Aufstand in La Bisbal gelingt, die beiden Regimenter werden vernichtet, nur der Leutnant von Jochberg überlebt - er wird nämlich von den Insurgenten für den Marques gehalten.

Dann nimmt der Roman eine überraschende Schlusswendung. Der Ich-Erzähler Leutnant von Jochberg betrachtet sich im Spiegel und stellt fest: „In mir war groß und furchtbar der Marques de Bolibar. [...] Und es war mir, als wäre die Vernichtung der Regimenter von Anfang an mein Wille gewesen, als hätte ich sie bei mir beschlossen gehabt, um einer großen und erhabenen Sache willen“" (Perutz 2006, S. 238). Der Besiegte verlässt La Bisbal in der Gestalt des Siegers mit der Feststellung: „Nicht ich, der tote Marques de Bolibar ging durch die Gassen seiner Stadt“ (Perutz 2006, S. 242). Diese paradoxe Feststellung löst nicht allein Zweifel an der Verlässlichkeit der Ich-Erzählung im Einzelnen aus, sondern an ihrer gesamten Raison d'être. Sie berichtet von der Niederlage der Rheinbundregimenter - aber was wollen die Memoiren des Leutnants von Jochberg eigentlich erzählen: die Geschichte seiner und seiner Offizierskameraden schmählichen Niederlage oder die Geschichte des Siegs des Marques de Bolibar?

Wie die Schlusswendung in Zwischen neun und neun lässt der final twist des Marques de Bolibar die gesamte Binnenerzählung schlagartig in einem anderen Licht erscheinen. Ließ der erstere Roman den Leser ratlos zurück über die

eine phantastische Erklärung gewählt würde, die Frage nach der Funktion dieser Verwandlung in den Memoiren des Leutnants von Jochberg immer noch ein Rätsel bleibt, vgl. Martínez (1996, S. 177-202). 
ontologische Qualität einer Erzählung, die aufgrund der Erzählweise Realität nicht sein wollte und Traum nicht sein konnte, so bleibt der Marques de Bolibar der Leserin nicht weniger als den Sinn der erzählten Geschichte schuldig. Auf der Suche nach ihm erweist sich der Herausgeber der Memoiren als ausgesprochen unkooperativ: Ihn interessieren nicht die Motive des Leutnants von Jochberg, sondern allein die Glaubwürdigkeit von dessen Memoiren, die er in der Rolle des professionellen Historikers ironisch in Zweifel zieht. Offen bleibt, warum der Historiker sie gleichwohl ediert - und sie sogar noch um ein Drittel kürzt - in der Meinung die Memoiren hätten dadurch ,an Wirkung und innerer Spannkraft gewonnen“ (Perutz 2006, S. 10).

Perutz' nächster Roman, Der Meister des Jüngsten Tages, ist ein Gegenwartsroman, der im Wien des Jahres 1909 spielt. Konzeptionell knüpft er an den Marques de Bolibar insofern an, als die Schlusswendung hier wie dort eine erneute Lektüre des Romans oder zumindest eine Reinterpretation der Erstlektüre nahelegt. Beim Meister des Jüngsten Tages handelt es sich um eine asymmetrische Rahmenerzählung, die eine autodiegetische Binnenerzählung einfasst. Das „Vorwort statt eines Nachworts" setzt ein mit einer Versicherung des Freiherrn von Yosch, er wolle die traumatischen und tragischen Ereignisse um den Tod des Hofschauspielers Eugen Bischoff wahrheitsgemäß schildern. Doch an die Stelle eines nüchternen Berichts treten schon im Vorwort dunkle Hinweise auf eine „Verfolgung“, die einem furchtbaren „Revenant aus vergangenen Jahrhunderten“ (Perutz 2003, S. 8) galt. Die Binnenerzählung des autodiegetischen Erzählers Yosch beginnt hingegen nüchtern und liest sich wie ein Kriminalroman. Zwei Freunde des Freiherrn klären den Mord an Eugen Bischoff als Glied einer Mordserie auf: Als ,Mörder' wird ein Foliant aus dem 16. Jahrhundert entlarvt, der das Rezept für eine letztlich tödlich wirkende halluzinogene Droge enthält; sie vermag die kreativen Kräfte von Künstlern zu stimulieren. Beendet wird der Roman durch „Schlußbemerkungen des Herausgebers“. Ein Regimentskamerad des Freiherrn teilt hier mit, dass Yoschs Aufzeichnungen nur im ersten Teil der Wahrheit entsprechen, dann aber ,mit einer jähen Wendung ins Phantastische“ (Perutz 2003, S. 196) abbiegen - in Wirklichkeit nämlich habe die Affäre ,,in einer ehrengerichtlichen Verurteilung des Freiherrn von Yosch ihren Abschluß gefunden“(Perutz 2003, S. 196). ${ }^{8}$ Was sich im Bericht des Freiherrn in einer ersten Lektüre als wahre Darstellung gelesen haben mag, kann in zweiter Lektüre als Fiktion über ein Trauma gelesen werden, die beseelt ist vom Drang des Schuldigen, die Indizien seiner Tat gewaltsam im Sinne seiner Unschuld umzudeuten (vgl. Perutz 2003, S. 197). Bei einer genaueren Lektüre kann der Leser indes feststellen, dass die Binnenerzählung nicht allein die Version der Leugnung der Schuld, sondern auch den Subtext des Eingeständnisses eben dieser Schuld enthält, die sich im eingeschobenen Bericht des „Pompeo di Bene“ kristallisiert. Als wohlkonstruierter Kompromiss zwischen Detektiv- und Künstlerroman lässt sich der autodiegetische

${ }^{8} \mathrm{Zu}$ den möglichen emotionalen Reaktionen auf diese Enthüllung vgl. Hillebrandt (2011, S. 224 f.). 
Bericht des Freiherrn sowohl als Leugnung wie auch als Beichte des Mords lesen. ${ }^{9}$ Dass er überdies noch einen Einblick in die Konstruktionsprinzipien des Entlastungsberichts freigibt, sei hier nur angemerkt. - Ohne Zweifel ist der Freiherr von Yosch ein unzuverlässiger Erzähler.

Die Romane Turlupin und Wohin rollst Du, Äpfelchen ... streife ich nur, weil sie - bei großen Unterschieden im Einzelnen - im Hinblick auf das unzuverlässige Erzählen und seine Funktion in Perutz' Entwicklung kaum Neues bieten. Hatte der Marques de Bolibar in der Binnenerzählung die Erklärung eines unerklärlichen Ereignisses geleistet, so bietet Turlupin in der Binnengeschichte die Erklärung für ein nicht stattgehabtes Ereignis; der Roman erklärt, wieso es im Jahre 1642 nicht zur französischen Revolution kommen konnte, sodass sie sich tatsächlich erst 1789 ereignete. Dieses knappe Resümee besagt selbstverständlich nicht, dass der Roman über den vom Schicksal auserkorenen Narren Turlupin nicht ebenso Spannung aufbaut und eine überraschende Schlusswendung enthält wie die zuvor erwähnten Romane.

Dasselbe gilt ähnlich auch für Wohin rollst Du, Äpfelchen ... einen Kolportageroman für die Berliner Illustrirte Zeitung. Der Roman hat eine auf das Massenpublikum berechnete vereinfachte Erzählstruktur: keinen Rahmen, kein Rätsel, die chronologische Erzählung einer spannenden Verfolgungsjagd mit vielen Überraschungen und einem final twist, der verblüfft, aber nicht zu einer Relektüre zwingt.

Ein zünftiger unzuverlässiger Ich-Erzähler erzählt den 1933 erschienenen Roman St. Petri Schnee. Mit der Dritten Kugel ist er verwandt, weil die Helden beider Romane wichtige Teile ihrer Lebensgeschichte vergessen haben und zu rekonstruieren suchen. Kann Hauptmann Glasäpflein hoffen, sie aus der Erzählung des spanischen Reiters zu erfahren, so muss sich Georg Friedrich Amberg, der autodiegetische Erzähler von St. Petri Schnee auf sein eigenes Gedächtnis verlassen. In technischer Hinsicht ist der Roman auch mit dem Meister des Jüngsten Tages verwandt. Bewirkt hier das „Nachwort des Herausgebers“ eine Lektüre, die von Yoschs Erzählung zugleich als Leugnungs- und Geständnisgeschichte zu lesen ermöglicht, so enthält Ambergs Geschichte von Beginn an zwei konkurrierenden Versionen, die seine Gedächtnislücke aufzufüllen vermögen. Gemäß dem vom Krankenhauspersonal gelieferten Bericht liegt Amberg nach einem in Osnabrück erlittenen Unfall seit etwa fünf Wochen im dortigen Krankenhaus; nach seiner eigenen Erinnerung hat er diese Zeit überwiegend in Morwede verbracht und befindet sich erst seit ein paar Tagen im Osnabrücker Krankenhaus. Die Krankenhaus- und die Morwede-Geschichte schließen einander aus - in Ambergs Erinnerungsprozess gibt es jedoch einzelne Fragmente, die in beide Geschichten zu passen scheinen: „der leise Geruch des Chloroforms“ (Perutz 2005, S. 81) und einige Personen, die, wenngleich in verschiedenen Funktionen, in beiden Geschichten vorzukommen scheinen.

\footnotetext{
${ }^{9}$ Vgl. dazu im Einzelnen Hillebrandt (2011, S. 218-230) und Beck (2017, S. 561-598).
} 
Die Morwede-Geschichte hat zwei Handlungskerne: zum einen die chemische Produktion eines Rauschmittels, das die Menschen in Morwede und Umgebung zum wahren Gottesglauben zurückführen und das Kaisertum des Staufer-Reichs restituieren soll, und zum anderen eine Liebesnacht mit der Chefchemikerin Bibiche. Amberg hält zäh an der Morwede-Geschichte fest; diese führt zwar nicht zu einer Repristination des Staufer-Reichs, sondern zu einem Pöbel-Aufstand, bei dem Amberg schwer verwundet wird, aber sie enthält doch zum Mindesten die Erinnerung an eine Liebesnacht, die Ambergs Arzt freilich nur als Wunschtraum qualifiziert. Amberg gelingt es mit allerlei Einfällen, sich gegen die Desillusionierung und die Zerstörung seines Traums zu immunisieren; er verlässt das Krankenhaus als glücklicher Mensch.

Damit haben wir auf alle Perutz-Romane mit einem unzuverlässigen Erzähler einen Blick geworfen. ${ }^{10}$ Die drei Romane, die Perutz nach St. Petri Schnee schrieb, haben alle einen zuverlässigen Erzähler. Der schwedische Reiter kann nur von einem auktorialen Erzähler erzählt werden, denn sein Held sind zwei Männer, die lediglich in der Erinnerung zu einer Gestalt verschmolzen sind. Nachts unter der steinernen Brücke ist ein vielschichtiges Gebilde, das aus disjunkten Episoden den Kern eines Romanplots zu generieren gestattet und in einer eigenwilligen Rahmenkonstruktion zugleich vorgibt, mündliche Erzählung zu sein. Im Hinblick auf die Komposition ist Perutz' letzter Roman, Der Judas des Leonardo, ganz das Gegenteil seines Vorgängers: ein Roman von verblüffender Schlichtheit. Ein böhmischer Kaufmann, der in Mailand seine Liebe zur schönen Niccola verrät, ein an Villon erinnernder lyrischer Dichter namens Mancino, der für seine unglückliche Liebe zu Niccola stirbt, und der entrückte Künstler Leonardo, dem zur Vollendung seines Abendmahls nur ein Vorbild für den Judas fehlt, das Mancino ihm in Gestalt Behaims liefert. Unter dieser Geschichte liegt freilich noch eine andere verborgen, die bislang nicht ans Licht gebracht ist.

Die Einteilung der Romane von Leo Perutz nach zuverlässigen beziehungsweise unzuverlässigen Erzählern ist eine retrospektive Klassifikation: kein zeitgenössischer Literaturkritiker hat sie vorgenommen; sie wurde erst durch die Narratologie in der Nachfolge von Wayne C. Booth ermöglicht. Die Zeitgenossen hielten Perutz' Romane zwar für Produkte einer exzeptionell ,ausgepichten Erzählbegabung“ (Polgar 1924, S. 508), aber genauere Beobachtungen zur Erzählweise stellten sie kaum an. Eine Ausnahme stellt allein der Roman Zwischen neun und neun dar, an dessen Erzählkonzeption mehrere Literaturkritiker Anstoß nahmen. Alfred Kerr machte auf die „offenbare Traumunmöglichkeit“ des durch den Romanschluss erwiesenen Traums aufmerksam und kritisierte: „In so erwogener Reihenfolge fiebert sichs kaum“ (Kerr 1923). Nicht nur Kerr hielt diese Konstruktion für einen „zweifelhaften Kunstgriff“, der allenfalls durch seine „unleugbar kräftige Wirkung“ (Wolf 1919, S. 432) legitimiert werden könne. Der mit Perutz befreundete Schriftsteller Ernst Weiß hingegen, selbst ein Meister im

\footnotetext{
${ }^{10}$ Die sich dieser Technik bedienenden Erzählungen von Perutz lasse ich hier außer Acht.
} 
Einsatz unzuverlässiger Erzähler, hielt Perutz' Roman Zwischen neun und neun für das „fabelhafteste, was ich gesehen habe“ (Weiß 1918, nach Perutz-Ausstellung 1989, S. 84). Dass die ,unmögliche' Traumerzählung im Roman nur durch einen unzuverlässigen Erzähler erfolgen konnte, merkte er nicht eigens an.

\section{Gibt es (nur) eine Poetik des unzuverlässigen Erzählens?}

Im ersten Jahrzehnt nach dem Zweiten Weltkrieg knüpfte in der deutschsprachigen Literatur niemand an Perutz' Erzählverfahren an. Perutz selbst aber hatte schon seit St. Petri Schnee (1933) keinen Gebrauch mehr vom unzuverlässigen Erzähler gemacht.

Gibt es dafür eine plausible Erklärung? Zum Ausgangspunkt der Suche nach einer triftigen Hypothese mag ein Zitat von Tom Kindt dienen, der in einer Untersuchung über Perutz' Roman Turlupin konstatierte:

Die Romane und Novellen von Leo Perutz nehmen im Zusammenhang der Literatur der Wiener und Prager Moderne zweifellos eine Sonderstellung ein. Gleichwohl weist eine Reihe seiner Texte zwei Merkmale auf, die kennzeichnend sind für nicht wenige Werke von Autoren des ,Jüngsten Wien' und der ,Prager deutschen Literatur' wie etwa Franz Kafka, Ernst Weiß, Franz Werfel oder Hermann Ungar. Das eine Merkmal ist ein inhaltliches: Perutz setzt sich in vielen seiner Romane und Novellen mit einer bestimmten Sicht der so genannten ,Krise des Ich' auseinander, er behandelt die Frage der Identität als Problem der Selbstannahme. Das andere Merkmal ist ein erzählerisches: Er bedient sich zur Gestaltung jenes Themas oftmals eines bestimmten Verfahrens, er nutzt Techniken erzählerischer Unzuverlässigkeit. (Kindt 2007, S. 77)

Kindts Feststellung impliziert vermutlich die These, dass das unzuverlässige Erzählen ein Merkmal der literarischen Moderne ist und diese These könnte man mit der - in der Literaturgeschichtsschreibung weit verbreiteten - These von der sich abschwächenden innovativen Kraft der Moderne ${ }^{11}$ in den Zwanzigerjahren und im Literarischen Exil verbinden und entsprechend behaupten, die nachlassende Kraft der literarischen Moderne habe sich in Perutz' Werk so ausgewirkt, dass er nach St. Petri Schnee vom unzuverlässigen Erzählen keinen Gebrauch mehr gemacht habe. Diese Hypothese ist nicht prinzipiell unplausibel, doch im Fall von Perutz sprechen schon gewichtige philologische Argumente gegen sie: Perutz hatte schon vor St. Petri Schnee mehrfach zuverlässig erzählte Romane geschrieben. ${ }^{12}$

\footnotetext{
${ }^{11}$ In der Literatur wird in diesem Zusammenhang auch von einer Transformation der Moderne, der Herausbildung einer „Reflektierten Moderne“ (Kiesel 2004, S. 299) einer Differenzierung oder der Herausbildung einer Tradition der Moderne gesprochen.

${ }^{12}$ Schon in Perutz' Erstling, der Dritten Kugel (1915), spielt das unzuverlässige Erzählen keine relevante Rolle, dasselbe gilt mindestens auch für Turlupin (1924). Ferner ist darauf hinzuweisen, dass Perutz bereits 1925 die erste Episode des Romans Nachts unter der steinernen Brücke publizierte, in dem es keinen unzuverlässige Erzähler gibt (s. Perutz 1924/1925, S. 247-256) und
} 
Aber mir scheint es grundsätzlich fraglich, ob es zwischen dem unzuverlässigen Erzählen und der literarischen Moderne mehr als einen möglicherweise rein statistischen Zusammenhang gibt. Wenn es einen konzeptionellen Zusammenhang gäbe: Wie ließe er sich beschreiben, wie könnte ein entsprechendes poetisches Programm aussehen? Auf welche Schwierigkeiten man bei seiner Formulierung stoßen würde, lässt sich schon in kleinstem Rahmen am Beispiel von Leo Perutz und Ernst Weiß zeigen, die einander kannten und zeitweise in derselben literarischen Gruppierung ${ }^{13}$ verkehrten.

Ernst Weiß' große Romane lassen sich dem von Matthias Aumüller herausgearbeiteten Typus des unzuverlässigen Erzählens mit offener Funktion zuordnen, einem Romantypus, bei dem sich die zahllosen Widersprüche des Erzählers nicht $\mathrm{zu}$ einer widerspruchsfreien Version integrieren lassen (vgl. Aumüller 2018, S. 143-146). Diese Erzählweise ist, so behaupte ich abkürzend, Bestandteil eines poetischen Programms, das den Leser auf strategische Weise kognitiv wie emotional desorientiert und verstört, um ihm nicht demonstrativ, sondern qua Lektüre erfahrungsgebunden nahezubringen, dass existentielle Verunsicherung sich nicht kognitiv bewältigen lässt. Ein poetisches Programm, wie Tom Kindt (2008) und Christiane Dätsch (2009) es aus Weiß' Essays rekonstruieren konnten, lässt sich bei Perutz nicht auffinden. Immerhin lässt sich aber empirisch feststellen, dass er im Gegensatz zu Weiß die geschlossene Funktion des unzuverlässigen Erzählens gewählt hat: In seinen Romanen ist von einem bestimmten Punkt der Erzählung an stets klar entscheidbar, was innerfiktional der Fall ist bzw. alternativ der Fall sein könnte. Die Entlarvung der Unzuverlässigkeit des Erzählers ist in Perutz' Romanen meist nur der trigger, der einen Rahmenwechsel ${ }^{14}$, einen frame-switch zur Rekonstruktion der Romanhandlung erforderlich macht: Nachdem eine Version der Erzählung als unglaubwürdig erwiesen ist, gilt es, eine neue, passendere zu suchen, deren Vorzüge und Mängel mit der vorigen abzugleichen sind usw. Die rekursive Suche nach einer oder nach der integrativen Rekonstruktion und Interpretation ist, so scheint mir, das eigentliche Ziel des Perutzschen Erzählprogramms ${ }^{15}$ : Rätsellösen nach Anhaltspunkten auf einer

schließlich hatte Perutz die Konzeption des Schwedischen Reiters, für den dasselbe gilt, bereits 1928 ausgearbeitet, bevor er mit St. Petri Schnee überhaupt begonnen hatte, vgl. dazu: PerutzAusstellung (1989. S. 214, 215, 234).

${ }^{13}$ Über den informellen literarischen Zirkel „Freilicht“ vgl. Müller (2007, S. 33-36).

${ }^{14}$ Auf die Bedeutung der ,Verstehensrahmen ‘ für die Interpretation von Perutz' Romanen hat in Anknüpfung an Erving Goffman zuerst Matías Martínez (2002) hingewiesen. - In der kognitiven Narratologie und Stilistik spielt die Entwicklung und Auswechslung (,frame switch“) von mentalen Modelle oder frames einer Erzählung eine zentrale Rolle, vgl. dazu etwa Emmott (1997, S. 269) und ausführlich Burke (2011) - Vgl. auch den verwandten Begriff des "narrative schema" bei Branigan (1992, S. 13 f.).

${ }^{15}$ Diese Feststellung gilt bereits für Die dritte Kugel, aber auch noch für den Roman Nachts unter der steinernen Brücke, in dem die Leserin stets zwischen dem plot der einzelnen Novellen und dem in statu nascendi befindlichen Romanplot hin und her manövrieren muss. 
Spielvorlage. Will man das Ziel der Romanpoetik von Ernst Weiß als ein ethisches bezeichnen, so kann man das von Perutz als ein hedonistisches ${ }^{16}$ charakterisieren: Literatur ist kein Weg zum besseren Leben, sondern zum besseren, lust- und genussvolleren Lesen und zur Erkenntnis von dessen Voraussetzungen - Metafiktion. Dazu braucht es Romankonstruktionen von großer Präzision und Eleganz, bei der neben allen anderen von Perutz selbstverständlich auch bedienten diegetischen ${ }^{17}$ Emotionen die Artefaktemotionen ${ }^{18}$ eine herausragende Rolle spielen.

Als vorsichtiges Fazit würde ich aus dem Fall Perutz die Konsequenz ziehen, dass das unzuverlässige Erzählen in so verschiedenartigen Erzählformen realisiert werden kann, dass ihm weder ein „charakteristisches emotionales Wirkungspotenzial“" (Hillebrandt 2011, S. 235) noch ein spezifisches poetisches Programm zugerechnet werden kann - ein Fazit, das dem statistisch gehäuften Auftreten des unzuverlässigen Erzählens in der literarischen Moderne um 1900 nicht widerspricht.

\section{Literatur}

Aumüller, Matthias: Offenheit und Geschlossenheit als Funktionen des unzuverlässigen Erzählens. Mit Interpretationsbeispielen anhand von Texten von Ernst Weiß, Paul Zech und Stefan Zweig. In: Journal of Literary Theory 12.1 (2018), 126-149.

Beck, Sandra: Wiederholungen und fremde Autoren. Leo Perutz: Der Meister des Jüngsten Tages (1923). In: Narratologische Ermittlungen. Muster detektorischen Erzählens in der deutschsprachigen Literatur. Hg. Sandra Beck. Heidelberg: Winter, 2017, 561-598.

Branigan, Edward: Narrative Comprehension and Film. London: Routledge, 1992.

Burke, Michael: Literary Reading, Cognition and Emotion. An Exploration of the Oceanic Mind. New York, London: Routledge, 2011.

Dätsch, Christiane: Existenzproblematik und Erzählstrategie. Studien zum parabolischen Erzählen in der Kurzprosa von Ernst Weiß. Tübingen: Niemeyer, 2009 (= Studien zur deutschen Literatur 186).

Eder, Jens: Casablanca and the Richness of Emotion. In: Journal of Literary Theory 1.2 (2007), 231-250.

Emmott, Catherine: Narrative Comprehension. A Discourse Perspective. Oxford: Oxford University Press, 1997.

Hillebrandt, Claudia: Das emotionale Wirkungspotenzial von Erzähltexten: mit Fallstudien zu Kafka, Perutz und Werfel. Berlin: Akademie Verlag, 2011 (= Deutsche Literatur. Studien und Quellen 6).

\footnotetext{
${ }^{16}$ Weiß war, wie Tom Kindt dargestellt hat, ein scharfer Kritiker des Erzählprogramms von Perutz, obgleich er dessen Romane bewunderte, vgl. Kindt (2007, S. 69).

${ }^{17}$ Zum Terminus vgl. Eder (2007, S. 234 f.): „Developing mental models of a fictive world brings forth character- and situation-related feelings, in short, diegetic emotions that concern the represented world (diegesis).“

${ }^{18} \mathrm{Zu}$ Artefaktemotionen vgl. Tan (1996, S. 65) und Hillebrandt (2011, S. 134), mit Bezug auf Perutz ebd., S. 216, 225 u. ö. - Über das Zusammenwirken von diegetischen mit ArtefaktEmotionen vgl. Levinson (1997, S. 32) und ausführlicher Polvinen (2013, S. 165-180).
} 
Kerr, Alfred: Leo Perutz und Hans Sturm: Zwischen neun und neun. Theater in der Königgrätzer Straße. In: Berliner Tageblatt. Jg. 52 (1923), Nr. 593.

Kiesel, Helmuth: Geschichte der literarischen Moderne: Sprache, Ästhetik, Dichtung im zwanzigsten Jahrhundert. München: C.H.Beck, 2004.

Kindt, Tom: Turlupin oder: Und wo bleibt das Ethische, Herr Perutz? In: Leo Perutz' Romane Von der Struktur zur Bedeutung. Hgg. Tom Kindt und Jan Christoph Meister. Tübingen: De Gruyter, 2007, 69-80.

Kindt, Tom: Unzuverlässiges Erzählen und literarische Moderne. Eine Untersuchung der Romane von Ernst Weiß. Tübingen: Niemeyer, 2008 (= Studien zur deutschen Literatur 184).

Kindt, Tom und Tilmann Köppe: „Unreliable Narration with a Narrator and without“. In: Journal of Literary Theory 5.1 (2011), 81- 93.

Lamping, Dieter: Die fiktionale Sterbegeschichte. In: Von der Wachstafel zum Tonbandgerät. Vier Beiträge zur Literatur. Für Jürgen Born zum 60. Geburtstag. Wuppertal: Büro der Allgemeinen Literaturwissenschaft 1987 (= Wuppertaler Broschüre zur Allgemeinen Literaturwissenschaft 1), 71-95.

Levinson, Jerrold: Emotion in Response to Art. A Survey of the Terrain. In: Emotion and the Arts. Hgg. Mette Hjort und Sue Laver. Oxford: Oxford University Press, 1997, 20-36.

Martínez, Matías: Zwischen Apokalypse und Wahn. Leo Perutz, Der Marques de Bolibar (1920). In: Doppelte Welten, Struktur und Sinn zweideutigen Erzählens. Hg. Matías Martínez. Göttingen: Vandenhoeck \& Ruprecht 1996 (= Palaestra 298), 177-202.

Martínez, Matías: Proleptische Rätselromane. Erzählrahmen und Leserlenkung bei Leo Perutz. In: Leo Perutz. Unruhige Träume - Abgründige Konstruktionen. Dimension des Werks, Stationen der Wirkung. Hgg. Brigitte Forster und Hans-Harald Müller. Wien: Sonderzahl, 2002, 107-129.

Müller, Hans-Harald: Leo Perutz. Wien: Paul Zsolnay, 2007.

Ohme, Andreas: Skaz und Unreliable Narration. Entwurf einer neuen Typologie des Erzählers. Berlin u. a.: De Gruyter, 2015 (= Narratologia; 45).

Orosz, Magdolna: Erzählen - Identität - Erinnerung. Studien zur deutschsprachigen und ungarischen Literatur 1890-1935. Frankfurt am Main: Peter Lang, 2016 (= Budapester Studien zur Literatur 19), 305-331.

Perutz, Leo: Legende aus dem Ghetto. In: Der neue Merkur 8 (1924/25), 247-256.

Perutz, Leo: Der Meister des jüngsten Tages. München: dtv, 2003.

Perutz, Leo: Der Marques de Bolibar. München: dtv, 2006.

Perutz, Leo: St. Petri Schnee. München: dtv, 2005.

Perutz-Ausstellung 1989: Leo Perutz 1882-1957. Eine Ausstellung der Deutschen Bibliothek, Frankfurt am Main. Wien: Paul Zsolnay, 1989.

Polgar, Alfred: [Rez.: Leo Perutz:] Turlupin. In: Die Weltbühne 20 (1924), 506-508.

Polvinen, Merja: Affect and artifice in cognitive literary theory. In: Journal of Literary Semantics 42.2 (2013), 165-180.

Tan, Ed S.: Emotion and the Structure of Narrative Film. Film as an Emotion Machine. Mahwah, New Jersey: Routledge, 1996.

Titzmann, Michael: Das Konzept der ,Person“ und ihrer Identität in der deutschen Literatur um 1900. In: Die Modernisierung des Ich. Studien zur Subjektkonstitution in der Vor- und Frühmoderne. Hg. von Manfred Pfister. Passau: Wissenschaftsverlag Richard Rothe, 1989 (= PINK. Band 3), 36-52.

Titzman, Michael: ,Grenzziehung' vs. ,Grenztilgung'. Zu einer fundamentalen Differenz der Literatursysteme ,Realismus' und ,Frühe Moderne'. In: Weltentwürfe in Literatur und Medien. Phantastische Wirklichkeiten - realistische Imaginationen. Festschrift für Marianne Wünsch. Hgg. Hans Krah und Claus-Michael Orth. Kiel: Ludwig, 2002, 181-210.

Wolf, Hugo: [Rez.: Leo Perutz:] Zwischen neun und neun. In: Der Friede, Bd. 3, Nr. 70 (23. 5. 1919), 432. 
Open Access Dieses Kapitel wird unter der Creative Commons Namensnennung 4.0 International Lizenz (http://creativecommons.org/licenses/by/4.0/deed.de) veröffentlicht, welche die Nutzung, Vervielfältigung, Bearbeitung, Verbreitung und Wiedergabe in jeglichem Medium und Format erlaubt, sofern Sie den/die ursprünglichen Autor(en) und die Quelle ordnungsgemäß nennen, einen Link zur Creative Commons Lizenz beifügen und angeben, ob Änderungen vorgenommen wurden.

Die in diesem Kapitel enthaltenen Bilder und sonstiges Drittmaterial unterliegen ebenfalls der genannten Creative Commons Lizenz, sofern sich aus der Abbildungslegende nichts anderes ergibt. Sofern das betreffende Material nicht unter der genannten Creative Commons Lizenz steht und die betreffende Handlung nicht nach gesetzlichen Vorschriften erlaubt ist, ist für die oben aufgeführten Weiterverwendungen des Materials die Einwilligung des jeweiligen Rechteinhabers einzuholen. 